\title{
Investigation of the Required Discreteness of Interpolation Movement Parameters in Cyber-physical Systems
}

\author{
Volodymyr Kombarov¹, Yevgen Tsegelnyk ${ }^{2 *}$, Sergiy Plankovskyy³, Yevhen Aksonov¹, \\ Yevhen Kryzhyvets ${ }^{1}$
}

\begin{abstract}
1 Department of Automation and Computer-Integrated Technologies, Institute of Energy, Information and Transport Infrastructure, O. M. Beketov National University of Urban Economy in Kharkiv, 61002 Kharkiv, 17 Marshala Bazhanova Street, Ukraine

2 Department of Computer Science and Information Technology, Institute of Energy, Information and Transport Infrastructure, O. M. Beketov National University of Urban Economy in Kharkiv, 61002 Kharkiv, 17 Marshala Bazhanova Street, Ukraine

${ }^{3}$ Department of Physics, Institute of Energy, Information and Transport Infrastructure, O. M. Beketov National University of Urban Economy in Kharkiv, 61002 Kharkiv, 17 Marshala Bazhanova Street, Ukraine

* Corresponding author, e-mail: y.tsegelnyk@kname.edu.ua
\end{abstract}

Received: 19 January 2021, Accepted: 20 August 2021, Published online: 06 December 2021

\begin{abstract}
Improving the accuracy, reliability, and performance of cyber-physical systems such as high-speed machining, laser cutting, welding and cladding etc. is one of the most pressing challenges in modern industry. CNC system carries out data processing and significantly affect on accuracy of operation such equipment. The paper considers the problem of controlled axes motion differential characteristics data processing in the internal representation of the discrete space of the CNC system. Equations for determining the required discreteness of the differential characteristics position and resolution, such as the speed, acceleration, and jerk are proposed. For the most widely used CNC equipment specific discreteness and resolution values have been determined.
\end{abstract}

\section{Keywords}

CNC system, motion differential characteristics, speed, acceleration, jerk, feedforward, discreteness, resolution, high-speed machining, laser welding, laser cladding

\section{Introduction}

Modern technological equipment, which implements processes such as high speed machining [1, 2], laser cutting $[3,4]$, laser welding and cladding $[5,6]$, laser deburring $[7,8]$ etc. are cyber-physical systems. Such equipment must provide both high productivity and processing accuracy [9]. Providing smooth motion controlled axes equipment improves productivity and processing quality machined surface [10-13].

In Fig. 1 shown the generalized structure of a $\mathrm{CNC}$ system for high-speed equipment [14]. Each element of the system affects the accuracy of the process. Various parameters of CNC machine controls such as interpolation parameters, programming resolution or cycle times strongly influence the accuracy and dynamics of high precision axes [15-18].

The most widely the influence of trajectory shapes and feed control methods on accuracy and productivity are studied. In papers $[14,19-21]$ it was shown that the trajectories and laws of feedrate variation should provide a smooth variation of the motion differential characteristics such as speed, acceleration and jerk, taking into account the limitations of the controlled axes characteristics.

In number of papers [22-25] it was shown that the use of feedforward control taking into account the motion differential characteristics significantly increase the accuracy of movement along the tool path.

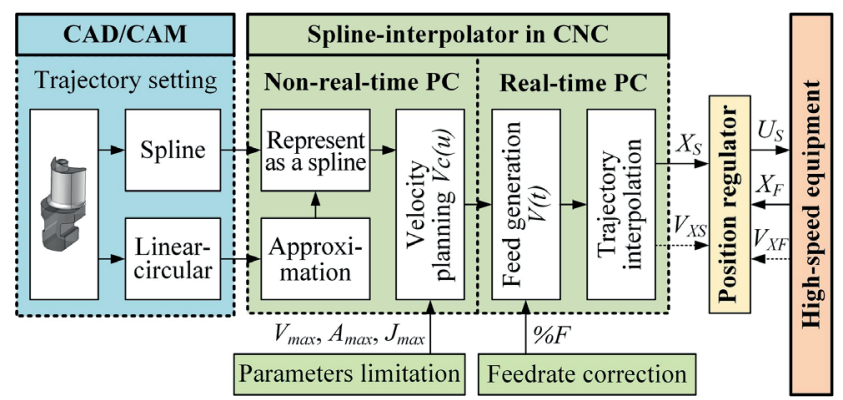

Fig. 1 Generalized structure of CNC system [14] 
In papers [26-29], various schemes of the CNC controllers are considered, in which feedforward control is used according to the differential characteristics of motion. The flow of position and speed commands is shown, but the requirements for these commands and the accuracy of setting the data that determine the position and speed of the controlled axis in the $\mathrm{CNC}$ are not specified.

Axes motion control is carried out in the discrete coordinate space of the machine at time clocking in accordance with a control cycle. Control parameters for each cycle are calculated in "Trajectory interpolation" block and used in the "Position regulator" block (Fig. 1). In case of insufficient discreteness occurs distortion of the differential kinematic characteristics in the internal representation of the parameters in the CNC system.

Determination of the required discreteness and resolution of representation position data of the controlled axes and their motion differential characteristics is an actual problem, providing the correct data processing for highspeed cyber-physical systems.

\section{Distortion of the controlled axes motion differential kinematic characteristics in a discrete representation of the CNC system parameters}

The use of feedforward control methods with speed and higher-order differential characteristics requires a corresponding resolution of control parameter [22-25]. Resolution should provide the calculating motion characteristics without any distortion. The control code is formed in the "Trajectory interpolation" block and transferred to the "Position regulator" block (Fig. 1). Typically, the input-output channel between these blocks has a limitation on the amount of transmitted data. As a rule, the information of the trajectory differential characteristics is not transmitted to the "Position regulator" block, and this information is necessary to restore by tool path finite differences [20]. Therefore, the control code passed from the "Trajectory interpolation" block to the "Position regulator" block must have necessary resolution to perform the calculations of the differential motion characteristics used to perform the feedforward control according to Eq. (1):

$$
\begin{aligned}
& V_{\text {axis }(i)}=\frac{X_{(i)}-X_{(i-1)}}{T_{\text {cycle }}}, a_{\text {axis }(i)}=\frac{V_{\text {axis }(i)}-V_{\text {axis }(i-1)}}{T_{\text {cycle }}}, \\
& J_{\text {axis }(i)}=\frac{a_{\text {axis }(i)}-a_{\text {axis }(i-1)}}{T_{\text {cycle }}},
\end{aligned}
$$

where $X_{(i)}, X_{(i-1)}$ are positions of the current and previous cycles; $V_{\text {axis }(i)}, V_{\text {axis }(i-1)}$ are velocities of the current and previous cycles; $a_{\text {axis }(i)}, a_{\text {axis }(i-1)}$ are accelerations of the current and previous cycles; $J_{\text {axis }(i)}$ is the jerk of the current cycle; $T_{\text {cycle }}$ is control cycle period.

The setpoint and actual effector position in the CNC system takes the form of integer code according to the position measurement discreteness. To avoid the accumulation of error of setpoint coordinate trajectory is not allowed to use a fractional representation of the position. The result of such limitations is insufficient resolution of the position control command data to calculate the motion differential characteristics. In addition, increasing the control cycle frequency leads to a decrease of the observed value of internal representation of speed and accordingly to the control deterioration. In Fig. 2 shown the results of speed and acceleration calculations according to the control axis acceleration area for the CNC system with a control cycle frequency of $1 \mathrm{kHz}$ and a discreteness of 1000 discrete/mm.

For the considered model at smooth feedrate $F_{\tau}$ variation Eq. (1) give discrete variation of speed $V_{\text {axis(i) }}$ and acceleration $a_{\text {axis }(i)}$. At the motion beginning the parameters of speed and acceleration "are not observed" and accordingly do not affect control. The appearance of interpolation values of speed and acceleration on the $35^{\text {th }}$ cycle forms the values at a level much higher than actually set, and the duration of these values is equal to one cycle. The equivalent frequency of the control code is $500 \mathrm{~Hz}$. This effect is outside the bandwidth of the servo drive electromechanical part and due to its filtering properties has no significant effect on the control process. This fact negatively affects the accuracy of the demand movements.
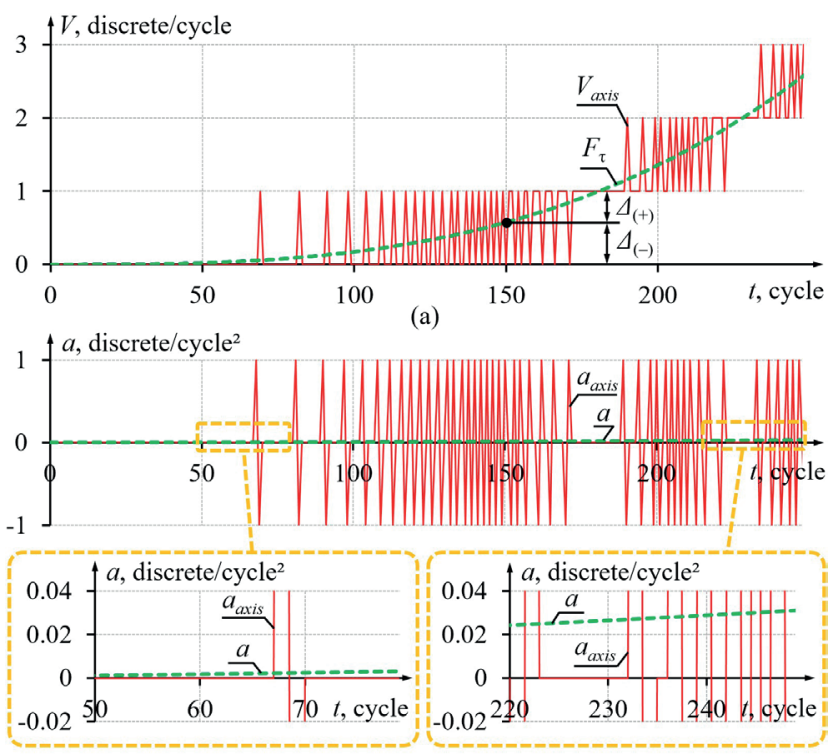

(b)

Fig. 2 Example of data calculations in the smooth acceleration area according to the axis position: (a) - speed $V_{a x i s} ;(\mathrm{b})-$ acceleration $a_{a x i s}$ 
An error of speed determination $\Delta_{(-)}, \Delta_{(+)}$in case of the movement with a fractional value of the internal representation of speed always will be present (Fig. 2). The relative error of the technological system controlled axes speed in this case is determined by Eq. (2):

$\Delta F_{\%}=\left(\frac{F_{\tau}-V_{(i)}}{F_{\tau}}\right) \times 100$.

The graph of the interpolation feedrate relative error is shown in Fig. 3. With feedrate $10 \mathrm{~mm} / \mathrm{min}$ the error is $+500 \%$
$-100 \%$ , and with $5000 \mathrm{~mm} / \mathrm{min}$ is $\pm 1 \%$ and slightly decreases with further feedrate increase. This error does not depend on the trajectory shape and will exist for all types of interpolation. The error is a characteristic of the position interpolation offset setpoint model.

Thus, the considered example demonstrates the distortion of differential characteristics, such as speed and acceleration in the internal representation according to the position discreteness. An increase in the resolution of data representation when processing control commands will provide the calculation of differential characteristics without their distortion.

\section{Determination of values ranges and required} resolution of controlled axes movement kinematic parameters in $\mathrm{CNC}$ systems

\subsection{Determination of the required position discreteness}

Graduation value of the machine axes position measuring determines the discreteness of the CNC system. The choice of necessary discreteness depends from the kinematic scheme of the controlled axis movement, type of the position sensor and a class of equipment accuracy.

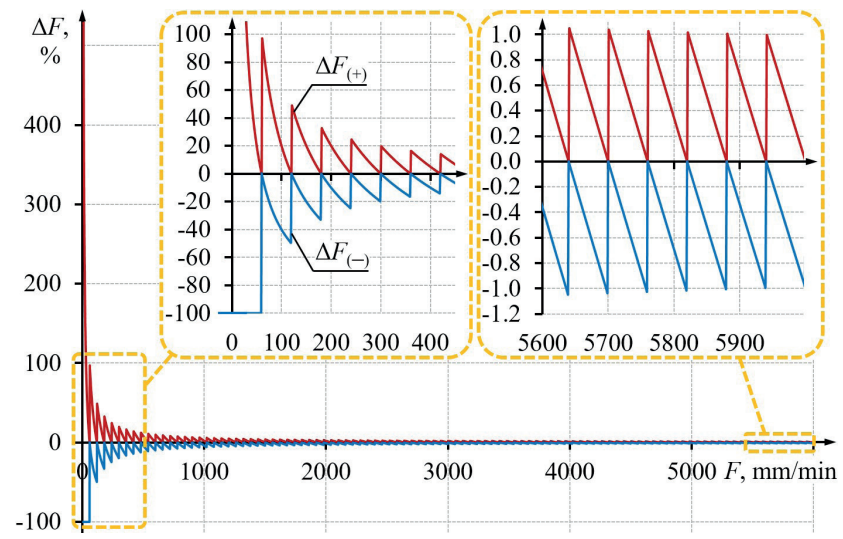

Fig. 3 Interpolation feedrate relative error for internal representation of speed: $\Delta F_{(+)}$is the error when integer representation is more than speed actual value; $\Delta F_{(-)}$is the error in the case of rounding to a value less than the speed actual value
Determination of the required discreteness (graduation value) of position measurement $\Delta X_{\min }$ is carried out depending on the standard tolerance grade the characteristic dimension of the part by Eq. (3):

$$
\Delta X_{\min }=\frac{P_{\text {accur }}}{K_{\text {measur }} \times 1000}
$$

where $P_{\text {accur }}$ is the standard tolerance grade of the part characteristic dimension, $\mu \mathrm{m} ; K_{\text {measur }}$ is the coefficient of increase discreteness of position measurement.

As a rule, the accuracy of a CNC machine should be four times higher than the specified accuracy of the part, therefore position measurement must be performed with increased discretization [30]. In this paper, the coefficient of increasing the discreteness of measuring the position in relation to the accuracy of the part $K_{\text {measur }}$ is taken equal 10 .

The order of the required discreteness and accordingly the discreteness coefficient of the position is determined by Eqs. (4) and (5):

$$
P D_{X}=\operatorname{int} \uparrow \lg \left(\frac{P_{\text {accur }}}{K_{\text {measur }} \times 1000}\right) \text {, }
$$

$k=10^{\left|P D_{X}\right|}$.

The results of required order discreteness $P D_{X}$ calculations depending on standard tolerance grade of parts machining with a characteristic dimension of the structural elements in the range of $10 \ldots 25 \mathrm{~mm}$ with the provision of the tolerance class defined in ISO 286-1:2010 [31] are shown in Fig. 4.

The overall dimensions of parts that are manufactured at machine-building plants are much larger than the selected characteristic dimension. A feature of the manufacture of such parts on $\mathrm{CNC}$ equipment is the need to ensure the accuracy of characteristic elements, and not the overall size. The vast majority of elements of such parts are made in the range of 10-7 standard tolerance grade. Accordingly, the most demanded from a technological point of view is equipment with a discreteness $10^{\left|P D_{X}\right|}$,

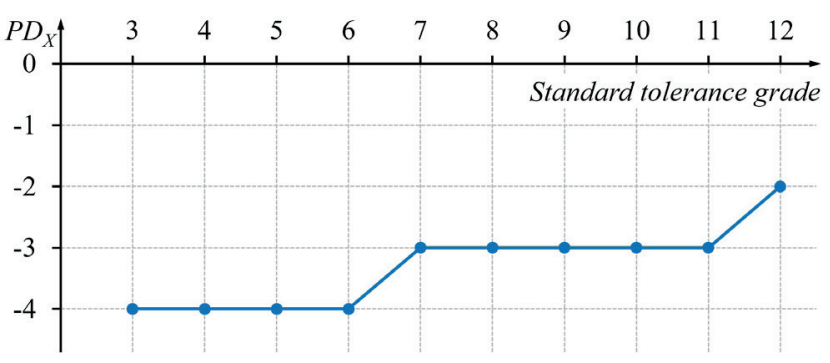

Fig. 4 The required order of position discreteness 
which is equal to $10^{-3} \mathrm{~mm}$. In addition, for the manufacture of parts with structural elements of 6-5 standard tolerance grade, order of $10^{-4} \mathrm{~mm}$ discreteness is required.

The obtained value of necessary discreteness corresponds to available position measurement systems parameters of the most widespread models of the machine-building enterprises equipment.

\subsection{Range of controlled axes motion parameters representation in the $\mathrm{CNC}$ system}

Conversion of motion kinematic characteristics such as speed, acceleration and jerk to the internal representation of the CNC system can be performed by Eqs. (6)-(8):

$$
\begin{aligned}
& V_{T}=\frac{F \times k}{f_{\text {cycle }} \times 60}, \\
& a_{T}=\frac{1000 \times a \times k}{f_{\text {cycle }}^{2}}=\frac{1000 \times n_{o v} \times g \times k}{f_{\text {cycle }}^{2}}, \\
& J_{T}=\frac{1000 \times J \times k}{f_{\text {cycle }}^{3}},
\end{aligned}
$$

where $F$ is the technological feedrate, $\mathrm{mm} / \mathrm{min} ; k$ is the discreteness of position measurement, discrete/mm; $f_{\text {cycle }}$ is the control cycle frequency, $\mathrm{Hz} ; n_{o v}$ is the overload factor; $g$ is the free fall acceleration, $\mathrm{m} / \mathrm{s}^{2}$.

In modern equipment designed for high-speed machining $\mathrm{CNC}$ systems with a control cycle frequency from 1 to $2.5 \mathrm{kHz}$ is usually used. In Fig. 5 shown the result of speed conversion for the corresponding range of the control cycle frequency and the most widely used discreteness $\left(10^{-3} \mathrm{~mm}\right)$. Small value of internal representation of speed $V_{T}$ corresponds to sufficiently large technological feedrates $F$. Therefore, at a control cycle frequency of $1 \mathrm{kHz}$, internal representation of speed value of 333 discrete/cycle corresponds to a technological feedrate of $20 \mathrm{~m} / \mathrm{min}$.

The speed of machine tool controlled axis movement in the traditional control model is defined by Eq. (1) as the finite difference of the position variation per time of control cycle. Due to the discreteness of the position measurement, a multiple of one discrete, a limited number of speed gradations can be recognized. In this example, for modern high-speed equipment it is possible to recognize only a few hundred speed gradations. At the same time, the depth of servo drives adjustment $K_{f}$ for the modern equipment makes at least $10000 \ldots 30000$. This means that the servo drive provides the machine tool controlled axis movement with the appropriate number of speed gradations. Obvious there is a discrepancy between the capabilities of the servo
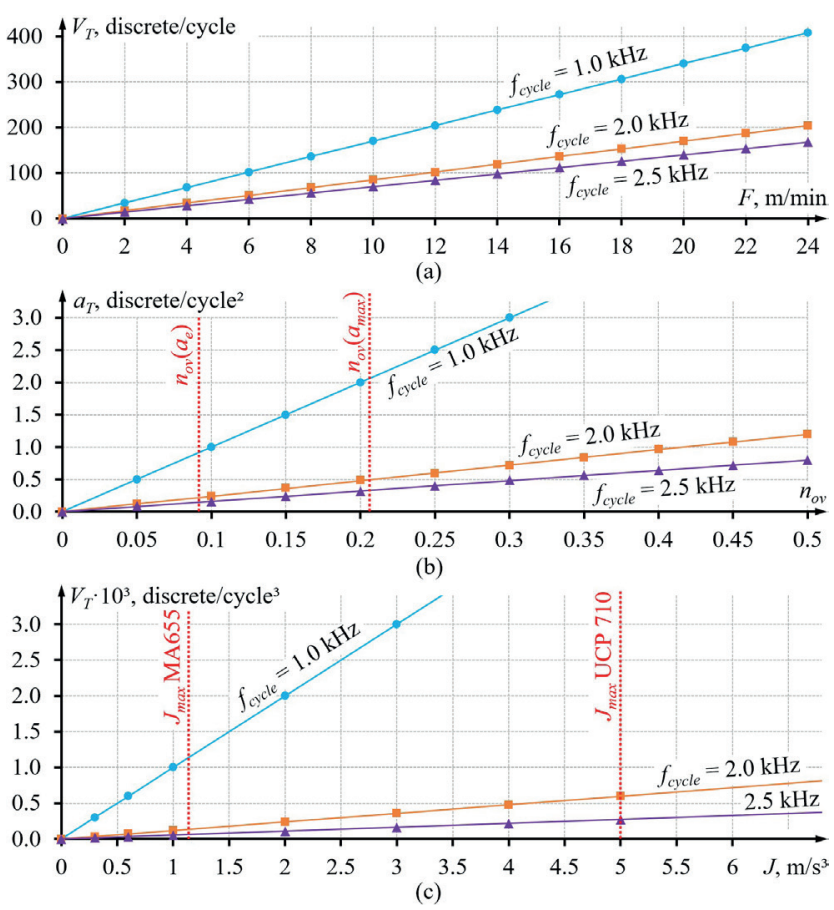

Fig. 5 Dependencies of motion parameters: (a) - internal representation of speed from technological feedrate; (b) - internal representation of acceleration from overload; (c) - internal representation of jerk from axis jerk; $a_{\max }$ - maximum acceleration; $a_{e}$ - operational acceleration; $J_{\max }$ - maximum jerk

drive to smoothly the speed variation in the processing system and the ability of the CNC system to observe the process of movement and control it accordingly. In this example, the CNC system determines the speed of the controlled axes from 100 to 300 times rougher than exist the ability to perform the movement. This means that to ensure correct observing of the movement and determine the physically realized speed of the machine tool controlled axis, it is necessary to increase the resolution to determine such a kinematic parameter as the internal representation of speed $V_{T}$ position variation.

The modern level of machine tool construction makes it possible to realize the acceleration of working bodies on milling machines tool of the order of $1.4 \ldots 2 \mathrm{~g}\left(14 \ldots 20 \mathrm{~m} / \mathrm{s}^{2}\right)$. For a CNC system with a control cycle frequency of $1 . .2 .5 \mathrm{kHz}$, such an overload corresponds to an internal representation of acceleration equal 19..3.14 discrete/cycle ${ }^{2}$. The maximum accelerations for the equipment operated at the machine-building enterprises are much smaller, than the specified value. Therefore, at the step of control code variation at toolplate longitudinal movement of the machine tool 16K20F3 the maximum acceleration makes 2.1 discrete/cycle ${ }^{2}$ that corresponds to an overload of $0.21 \mathrm{~g}$ (restriction $a_{\max }$ in Fig. 5). Operation of this machine tool is carried out apply of the S-shaped law of acceleration/ 
deceleration with the maximum value of acceleration of the order of 0.9 discrete/cycle ${ }^{2}$ that makes about $0.09 \mathrm{~g}$ (restriction $a_{e}$ on Fig. 5). The initial acceleration of the S-shaped law can be of $0.002 \ldots 0.010$ discrete/cycle ${ }^{2}$.

Experimental investigation on the machine type MA655SM30 shown the value of the maximum jerk in the internal representation of the CNC system depending on the control cycle frequency is not more than $0.001 \ldots 0.000064$ discrete/cycle ${ }^{3}$.

For $X, Y$ axes of MIKRON UCP 710 with $J_{\max }=5 \mathrm{~m} / \mathrm{s}^{3}$ [20], the jerk in the internal representation of the CNC system is $0.005 \ldots 0.00032$ discrete/cycle ${ }^{3}$, and for $Z$ axis with $J_{\max }=50 \mathrm{~m} / \mathrm{s}^{3}$ respectively $0.05 \ldots 0.0032$ discrete $/$ cycle $^{3}$.

Thus, it can be stated that in the internal representation of the CNC system, the differential characteristics of motion are the following order: speed are hundreds of discrete/cycle; acceleration are units or tenths of discrete/cycle ${ }^{2}$; jerk are hundredths or ten-thousandths of a discrete/cycle ${ }^{3}$.

The obtained estimate of the motion differential characteristics values in the internal representation of the $\mathrm{CNC}$ system allows us to assert that the discreteness of position determination, which meets the requirements of positioning accuracy, does not provide sufficient resolution for the speed parameter by approximately $100 \ldots 500$ times and has insufficient resolution to describe the parameters of acceleration and jerk. This circumstance excludes the possibility of smooth control of the axes movement with speed, acceleration and jerk feedforward.

\subsection{Determination of the required speed resolution}

To match the characteristics of the CNC system with the capabilities of the servo drive on the parameter of the depth of adjustment speed $K_{f}$, we determine the minimum required value of the speed in internal representation $\Delta F_{\min }$, which must be recognized by the CNC system:

$$
\Delta F_{\min }=\frac{F_{\max }}{60 \times f_{\text {cycle }} \times K_{f}},
$$

where $F_{\max }$ is the maximum permissible working feedrate, $\mathrm{mm} / \mathrm{min}$.

The order of the required speed resolution of the technological system controlled axes movement is calculated by Eq. (10):

$$
P D_{F}=\operatorname{int} \uparrow \lg \left(\frac{F_{\max }}{60 \times f_{\text {cycle }} \times K_{f}}\right) .
$$

To determine the degree of discrepancy between the required resolution and the available discreteness of position measurement, we write Eq. (11) for calculating the lowest observed speed $\Delta F_{\text {observ }}$ when it is determined from the position variation:

$$
\Delta F_{\text {observ }}=\frac{1}{k} \text {. }
$$

The ratio of the lowest observed feedrate $\Delta F_{\text {observ }}$ to the minimum required value of the speed in internal representation $\Delta F_{\min }$ shows the degree of discrepancy between the required resolution and the available discreteness of the position measurement:

$$
D_{F}=\frac{60 \times f_{\text {cycle }} \times K_{f}}{k \times F_{\max }} .
$$

In Fig. 6 shown the results of the required speed resolution calculations of the technological system controlled axes in a wide range of technological feedrates. Calculated for two system variants: one with control cycle frequency $1 \mathrm{kHz}$ and depth of adjustment servo drive $1 \times 10^{4}$, the other with control cycle frequency $2.5 \mathrm{kHz}$ and depth of adjustment servo drive $3 \times 10^{4}$.

Obviously, for the existing equipment of machine-building enterprises with a maximum feedrate of about $5 \mathrm{~m} / \mathrm{min}$, the version of the system with a control cycle frequency of $1 \mathrm{kHz}$ has a degree of inconsistency $D_{F}=300 \ldots 100$. In this case, the required speed resolution is $1 \times 10^{-5} \ldots 3 \times 10^{-6} \mathrm{~mm}$.

For equipment with a maximum feedrate of about $30 \mathrm{~m} / \mathrm{min}$ and a cycle frequency of $2.5 \mathrm{kHz}$, it has a degree of inconsistency $D_{F}=180$. In this case, the required speed resolution is $1 \times 10^{-5} \mathrm{~mm}$.

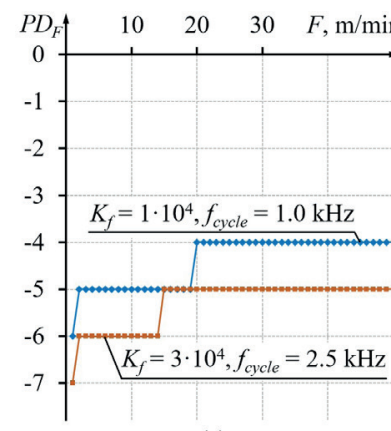

(a)

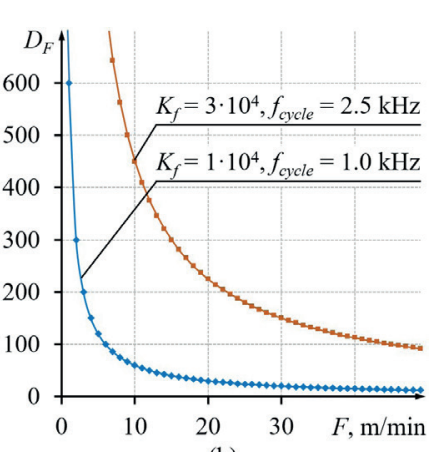

(b)
Fig. 6 Required speed resolution: (a) - order of the speed resolution depending on the feedrate; (b) - the degree of inconsistency between the required resolution and the available discreteness 


\subsection{Determination of the required acceleration resolution}

For specific equipment the lowest observed value of acceleration $\Delta a_{\min }$ can be calculated by Eq. (13):

$\Delta a_{\min }=\frac{1000 \times n_{o v} \times g}{f_{c y c l e}^{2} \times K_{a}}$,

where $K_{a}$ is the dimensionless coefficient of depth of adjustment acceleration.

To ensure sufficient smoothness of the acceleration variation the coefficient $K_{a}$ should be taken at least 1000 .

The order of the required acceleration resolution of the technological system movement can be calculated by Eq. (14):

$P D_{a}=\operatorname{int} \uparrow \lg \left(\frac{1000 \times n_{o v} \times g}{f_{\text {cycle }}^{2} \times K_{a}}\right)$.

To determine the degree of discrepancy between the required acceleration resolution and the available discreteness of the position measurement, we write an equation for calculating the smallest observed acceleration $\Delta a_{\text {observ }}$ by finite difference Eq. (1):

$\Delta a_{\text {observ }}=\frac{1}{k}$

The ratio of the lowest observed acceleration $\Delta a_{\text {observ }}$ to the minimum required value of the internal representation of acceleration shows the degree of discrepancy between the required acceleration resolution and the available discreteness of the position measurement:

$D_{a}=\frac{f_{c y c l e}^{2} \times K_{a}}{1000 \times n_{o v} \times g \times k}$.

The calculating results of the required resolution depending on the maximum acceleration for two variants of CNC systems with a control frequency of 1 and $2.5 \mathrm{kHz}$ and graphs of the discrepancy degree between the required acceleration resolution and the available discreteness are shown in Fig. 7.

To determine the acceleration parameters, it is necessary to provide a resolution of the order $1 \times 10^{-6} \ldots 1 \times 10^{-8} \mathrm{~mm}$ for equipment with a maximum acceleration characteristic of the order $0.2 \mathrm{~g}$.

\subsection{Determination of the required jerk resolution}

For specific equipment the smallest observed value of the jerk $\Delta J_{\min }$, can be calculated by Eq. (17):

$\Delta J_{\min }=\frac{1000 \times J_{\max }}{f_{\text {cycle }}^{3} \times K_{J}}$,

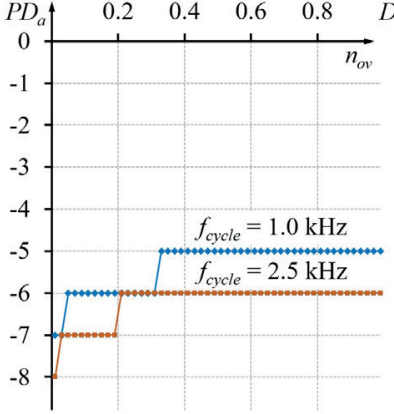

(a)

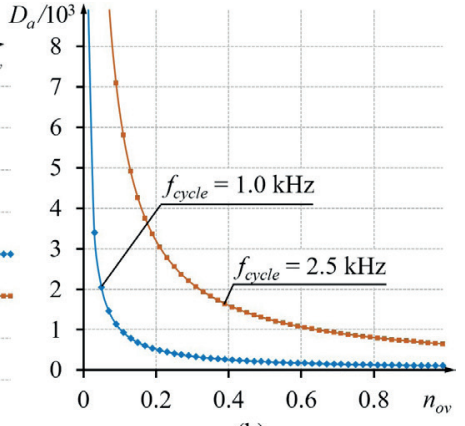

(b)
Fig. 7 Required acceleration resolution: (a) - order of the acceleration resolution depending on the overload; (b) - the degree of inconsistency between the required resolution and the available discreteness

where $J_{\max }$ is the maximum allowable jerk, $\mathrm{m} / \mathrm{s}^{3} ; K_{J}$ is the dimensionless coefficient of depth of adjustment jerk.

To ensure the minimum required smoothness of the jerk variation the coefficient $K_{J}$ should be taken at least 100 .

The order of the required jerk resolution of the technological system organs movement is calculated as follows:

$P D_{J}=\operatorname{int} \uparrow \lg \left(\frac{1000 \times J_{\max }}{f_{\text {cycle }}^{3} \times K_{J}}\right)$.

To determine the degree of discrepancy between the required jerk resolution and the available discreteness, we write an equation for calculating the smallest observed jerk $\Delta J_{\text {observ }}$ by the finite difference Eq. (1):

$\Delta J_{\text {observ }}=\frac{1}{k}$.

The ratio of the smallest observed jerk $\Delta J_{\text {observ }}$ to the minimum required value of the internal representation of jerk $\Delta J_{\min }$ shows the degree of discrepancy between the required jerk resolution and the available discreteness of the position measurement

$D_{J}=\frac{f_{\text {cycle }}^{3} \times K_{J}}{1000 \times J_{\max } \times k}$.

The calculating results of the required resolution depending on the maximum jerk for two variants of CNC systems with a control cycle frequency of 1 and $2.5 \mathrm{kHz}$ and graphs of the discrepancy degree between the required jerk resolution and the available discreteness are shown in Fig. 8.

To determine the jerk parameters it is necessary to provide a resolution of the order $1 \times 10^{-7} \ldots 1 \times 10^{-8} \mathrm{~mm}$ for equipment with a maximum jerk characteristic of the $1 \mathrm{~m} / \mathrm{s}^{3}$ and the depth of the adjustment jerk of 100 . With increasing depth of adjustment jerk to 1000 resolution should be increased to $1 \times 10^{-9} \mathrm{~mm}$. 


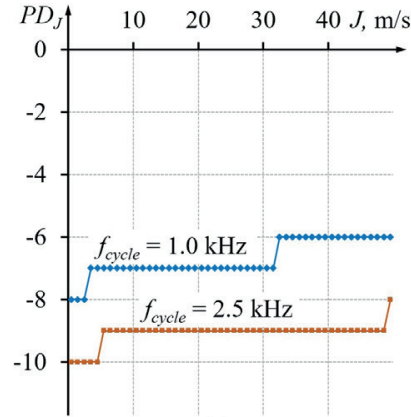

(a)

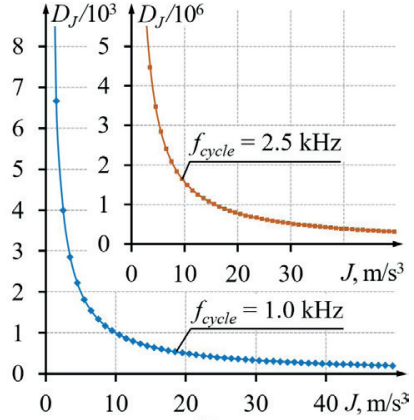

(b)
Fig. 8 Required jerk resolution: (a) - order of the jerk resolution depending on the maximum jerk; (b) - the degree of inconsistency between the required resolution and the available discreteness

\section{Conclusion}

The paper considers the problem of representing data of controlled axes motion differential characteristics for highspeed CNC equipment. As part of the motion control model in a discrete space with the time clocking in accordance with a control cycle obtained equations to determine the required position discreteness of controlled axes. The equations for evaluating the necessary resolution for feedforward control considering differential motion characteristics such as speed, acceleration and jerk are proposed.

A method for determining the required position discreteness and resolution of differential characteristics is proposed. The method takes into account the range of maximum feeds, the cycle frequency of the controller (sampling loop frequency), and the requirements for the accuracy of the technological system operation.

The proposed method allows determining the specific requirements for the controllers of technological systems.

\section{References}

[1] Permyakov, A., Dobrotvorskiy, S., Dobrovolska, L., Basova, Y., Ivanova, M. "Computer Modeling Application for Predicting of the Passing of the High-Speed Milling Machining Hardened Steel", In: Ivanov, V., Rong, Y., Trojanowska, J., Venus, J., Liaposhchenko, J. Z., Zajac, J., ..., Perakovic, D. (eds.) Advances in Design, Simulation and Manufacturing, Springer, Cham, Switzerland, 2019, pp. 135-145. https://doi.org/10.1007/978-3-319-93587-4_15

[2] Plankovskyy, S., Myntiuk, V., Tsegelnyk, Y., Zadorozhniy, S., Kombarov, V. "Analytical Methods for Determining the Static and Dynamic Behavior of Thin-Walled Structures During Machining", In: Shkarlet, S., Morozov, A., Palagin, A. (eds.) Mathematical Modeling and Simulation of Systems (MODS'2020), Springer, Cham, Switzerland, 2021, pp. 82-91. https://doi.org/10.1007/978-3-030-58124-4_8

[3] Anghel, C., Gupta, K., Jen, T. C. "Analysis and optimization of surface quality of stainless steel miniature gears manufactured by $\mathrm{CO}_{2}$ laser cutting", Optik, 203, Article number: 164049, 2020. https://doi.org/10.1016/j.ijleo.2019.164049

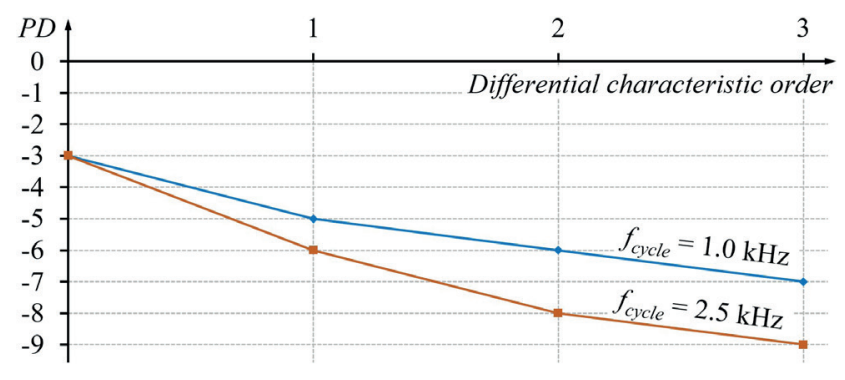

Fig. 9 The required resolution depending on the order of the differential kinematic characteristic

In Fig. 9 shown the dependence of the required resolution $P D$ on the order of the differential characteristic which can be used in the controlled axes movements process.

As a result of the research carried out, the necessary position discreteness and motion kinematic characteristics resolution of high-speed $\mathrm{CNC}$ equipment controlled axes was established. It is shown that in the processing of axes position data for precision positioning it is sufficient to use a discreteness of the order $1 \times 10^{-3} \ldots 3 \times 10^{-4} \mathrm{~mm}$.

The necessary resolution for the motion differential characteristics determining has been substantiated: for the speed determining it is necessary to ensure a resolution of the order $1 \times 10^{-5} \ldots 3 \times 10^{-6} \mathrm{~mm}$; for acceleration a resolution of the order $1 \times 10^{-6} \ldots 1 \times 10^{-8} \mathrm{~mm}$; and for jerk a resolution of the order $1 \times 10^{-7} \ldots 1 \times 10^{-9} \mathrm{~mm}$.

\section{Acknowledgement}

This research is supported by the Ministry of Education and Science of Ukraine as a part of the scientific research project No. 0121U109639.

[4] Plankovskyy, S., Tsegelnyk, Y., Shypul, O., Pankratov, A., Romanova, T. "Cutting Irregular Objects from the Rectangular Metal Sheet", In: Nechyporuk, M., Pavlikov, V., Kritskiy, D. (eds.) Integrated Computer Technologies in Mechanical Engineering, Springer, Cham, Switzerland, 2020, pp. 150-157.

https://doi.org/10.1007/978-3-030-37618-5_14

[5] Bernatskyi, A. V., Berdnikova, O. M., Klochkov, I. M., Sydorets, V. M., Chinakhov, D. A. "Laser Welding in Different Spatial Positions of T-joints of Austenitic Steel", IOP Conference Series: Materials Science and Engineering, 1(582), Article number: 012048, 2019.

https://doi.org/10.1088/1757-899X/582/1/012048

[6] Mahamood, R. M., Akinlabi, E. T., Owolabi, M. G. "Laser Metal Deposition Process for Product Remanufacturing", In: Gupta, K. (ed.) Advanced Manufacturing Technologies, Springer, Cham, Switzerland, 2017, pp. 267-291. https://doi.org/10.1007/978-3-319-56099-1_12 
[7] Cerwenka, G., Surrey, P., Möller, M., Conrad, C., Prakash, V., Heilemann, M., Emmelmann, C. "In-depth characterization of the scanner-based selective laser deburring process", Journal of Laser Applications, 30(3), Article number: 032510, 2018.

https://doi.org/10.2351/1.5040642

[8] Plankovskyy, S., Shypul, O., Tsegelnyk, Y., Tryfonov, O., Golovin, I. "Simulation of Surface Heating for Arbitrary Shape's Moving Bodies/Sources by Using R-functions", Acta Polytechnica, 56(6), pp. 472-477, 2016

https://doi.org/10.14311/AP.2016.56.0472

[9] Karpus, V., Ivanov, V., Dehtiarov, I., Zajac, J., Kurochkina, V. "Technological Assurance of Complex Parts Manufacturing", In: Ivanov, V., Rong, Y., Trojanowska, J., Venus, J., Liaposhchenko, O., Zajac, J., ..., Perakovic, D. (eds.) Advances in Design, Simulation and Manufacturing, Springer, Cham, Switzerland, 2019, pp. 51-61. https://doi.org/10.1007/978-3-319-93587-4_6

[10] Czampa, M., Biro, I., Szalay, T. "Effects of different cutting conditions on the surface roughness parameters of iron-copper-carbon powder metallurgy composites", International Journal of Machining and Machinability of Materials, 19(5), pp. 440-456, 2017. https://doi.org/10.1504/IJMMM.2017.087617

[11] Kolar, P., Sulitka, M., Matyska, V., Fojtu, P. "Optimization of Fiveaxis Finish Milling Using a Virtualmachine Tool", MM Science Journal, 2019(5), pp. 3534-3543, 2019. https://doi.org/10.17973/MMSJ.2019_12_2019037

[12] Vovk, A., Sölter, J., Karpuschewski, B. "Finite element simulations of the material loads and residual stresses in milling utilizing the CEL method", Procedia CIRP, 87, pp. 539-544, 2020 https://doi.org/10.1016/j.procir.2020.03.005

[13] Kurin, M. O. "Determination of the Boundaries of Plastic Zone of Metal Deformation During the Cutting", Progress in Physics of Metals, 21(2), pp. 249-273, 2020 https://doi.org/10.15407/ufm.21.02.249

[14] Kombarov, V., Sorokin, V., Fojtů, O., Aksonov, Y., Kryzhyvets, Y. "S-curve Algorithm of Acceleration/Deceleration with Smoothlylimited Jerk in High-speed Equipment Control Tasks", MM Science Journal, 2019(4), pp. 3264-3270, 2019. https://doi.org/10.17973/MMSJ.2019_11_2019080

[15] Brecher, C., Lindemann, D., Zavelberg, M., Wenzel, C. "Analysis of Control and Servo Drive Systems for the Application in Ultra Precision Machining", In: The $11^{\text {th }}$ euspen International Conference, Como, Italy, 2011.

[16] Sokolov, V., Krol, O., Stepanova, O. "Choice of Correcting Link for Electrohydraulic Servo Drive of Technological Equipment", In: Ivanov, V., Trojanowska, J., Machado, J., Liaposhchenko, O., Zajac, J., Pavlenko, I., ..., Perakovic, D. (eds.) Advances in Design, Simulation and Manufacturing II, Springer, Cham, Switzerland, 2020, pp. 702-710

https://doi.org/10.1007/978-3-030-22365-6_70

[17] Fesenko, A., Basova, Y., Ivanov, V., Ivanova, M., Yevsiukova, F., Gasanov, M. "Increasing of Equipment Efficiency by Intensification of Technological Processes", Periodica Polytechnica Mechanical Engineering, 63(1), pp. 67-73, 2019.

https://doi.org/10.3311/PPme.13198
[18] Smyrnyi, M. F., Pliuhin, V. Y., Polivianchuk, A. P., Gokov, A. M "Development of information devices for control of objects with a discrete mode of motion on the basis of magnetometric converters", Telecommunications and Radio Engineering, 79(19), pp. 1735-1746, 2020. https://doi.org/10.1615/TelecomRadEng.v79.i19.60

[19] Erkorkmaz, K., Altintas, Y. "High speed CNC system design. Part I: jerk limited trajectory generation and quintic spline interpolation", International Journal of Machine Tools and Manufacture, 41(9), pp. 1323-1345, 2001 https://doi.org/10.1016/S0890-6955(01)00002-5

[20] Beudaert, X., Lavernhe, S., Tournier, C. "Feedrate interpolation with axis jerk constraints on 5-axis NURBS and G1 tool path", International Journal of Machine Tools and Manufacture, 57, pp. 73-82, 2012.

https://doi.org/10.1016/j.ijmachtools.2012.02.005

[21] Kombarov, V., Sorokin, V., Tsegelnyk, Y., Plankovskyy, S., Aksonov, Y., Fojtů, O. "S-Shape Feedrate Scheduling Method with Smoothly-Limited Jerk in Cyber-Physical Systems", In: Cioboată, D.D. (ed.) International Conference on Reliable Systems Engineering (ICoRSE) - 2021, Springer, Cham, Switzerland, 2022, pp. 54-68. https://doi.org/10.1007/978-3-030-83368-8 6

[22] Conway, J. R., Darling, A. L., Ernesto, C. A., Farouki, R. T., Palomares, C. A. "Experimental study of contouring accuracy for $\mathrm{CNC}$ machines executing curved paths with constant and curvature-dependent feedrates", Robotics and Computer-Integrated Manufacturing, 29(2), pp. 357-369, 2013. https://doi.org/10.1016/j.rcim.2012.09.006

[23] Schraeder, T. F., Farouki, R. T. "Experimental performance analysis of an inverse dynamics CNC compensation scheme for highspeed execution of curved toolpaths", The International Journal of Advanced Manufacturing Technology, 73(1-4), pp. 195-208, 2014. https://doi.org/10.1007/s00170-014-5720-Z

[24] Aksonov, Y., Kombarov, V., Fojtů, O., Sorokin, V., Kryzhyvets, Y. "Investigation of Processes in High-speed Equipment Using CNC Capabilities", MM Science Journal, 2019(4), pp. 3271-3276, 2019. https://doi.org/10.17973/MMSJ.2019_11_2019081

[25] Kombarov, V., Sorokin, V., Tsegelnyk, Y., Plankovskyy, S., Aksonov, Y., Fojtů, O. "Numerical Control of Machining Parts from Aluminum Alloys with Sticking Minimization", International Journal of Mechatronics and Applied Mechanics, 1(9), pp. 209-216, 2021. https://oi.org/10.17683/ijomam/issue9.30

[26] Moscrop, J. W. "Modelling, analysis and control of linear feed axes in precision machine tools", $\mathrm{PhD}$ Thesis, University of Wollongong, 2008. [online] Available at: http://ro.uow.edu.au/ theses/27 [Accessed: 02 August 2021]

[27] Alkafafi, L. "Time and frequency optimal motion control of CNC machine tools", PhD Thesis, Technische Universität Hamburg, 2013. https://doi.org/10.15480/882.1102

[28] Lin, C. Y., Lee, C. H. "Remote Servo Tuning System for Multi-Axis CNC Machine Tools Using a Virtual Machine Tool Approach", Applied Sciences, 7(8), Article number: 776, 2017. https://doi.org/10.3390/app7080776 
[29] Jia, Z., Ma, J., Song, D., Wang, F., Liu, W. "A review of contouring-error reduction method in multi-axis $\mathrm{CNC}$ machining", International Journal of Machine Tools and Manufacture, 125, pp. 34-54, 2018.

https://doi.org/10.1016/j.ijmachtools.2017.10.008

[30] Wang, C. "Calibration of CNC machine tools", Aerospace Engineering, 8, pp. 10-11, 2006. [online] Available at: https:// www.optodyne.it/inglese/articles/AEaug06new.pdf [Accessed: 02 August 2021]
[31] International Organization for Standardization "ISO 286-1:2010 Geometrical product specifications (GPS) - ISO code system for tolerances on linear sizes - Part 1: Basis of tolerances, deviations and fits", International Organization for Standardization (ISO), Geneva, Switzerland, 2010. [online] Available at: https://www.iso. org/standard/45975.html [Accessed: 02 August 2021] 